\title{
The effect of toroidal plasma rotation on low-frequency reversed shear Alfvén eigenmodes in tokamaks
}

\author{
J W Haverkort ${ }^{1,2}$ \\ ${ }^{1}$ FOM Institute for Plasma Physics Rijnhuizen, Association EURATOM-FOM, Trilateral Euregio \\ Cluster, PO Box 1207, Nieuwegein, The Netherlands \\ ${ }^{2}$ Centrum Wiskunde \& Informatica (CWI), PO Box 94079, Amsterdam, The Netherlands \\ E-mail: J.W.Haverkort@cwi.nl
}

Received 15 July 2011, in final form 28 October 2011

Published 5 January 2012

Online at stacks.iop.org/PPCF/54/025005

\begin{abstract}
The influence of toroidal plasma rotation on the existence of reversed shear Alfvén eigenmodes (RSAEs) near their minimum frequency is investigated analytically. An existence condition is derived showing that a radially decreasing kinetic energy density is unfavourable for the existence of RSAEs. The Coriolis effect is typically unfavourable for modes moving slower than the plasma or moving in the opposite direction. The generality of the analysis allows for the description of downwards sweeping RSAEs and also rotation-induced modes in regular shear plasmas.
\end{abstract}

\section{Introduction}

To describe Alfvén waves associated with the bending of magnetic field lines in tokamak plasmas, the theory of magnetohydrodynamics (MHD) has been very successful. The continuous MHD spectrum contains various gaps and extrema introduced by toroidal effects, plasma shape and rotation. When specific criteria are met, global modes can cluster at these extrema. An example of such cluster modes are reversed shear Alfvén eigenmodes (RSAEs). They are often observed in magnetic spectrograms when the safety factor profile has an extremum. These modes have been used as an accurate diagnostic for the safety factor [1-3]. In addition to their usefulness in MHD spectroscopy, RSAEs can also have a negative effect on the confinement of energetic ions [4]. The expulsion of fast particles can lead to decreased plasma performance and even damage to plasma facing components.

RSAEs were first observed in JT-60U [5] and later thoroughly investigated at JET [2,6], where they were called Alfvén Cascades (ACs). They have also been observed in various other tokamaks such as Alcator C-mod [3], TFTR [7], ASDEX Upgrade [8], DIII-D [9] and spherical tokamaks such as NSTX [10] and MAST [11]. Recently, they have also been found in the LHD stellarator [12]. RSAEs are observed to terminate at the geodesic acoustic mode (GAM) frequency, primarily caused by geodesic curvature [13-15]. When this minimum frequency exceeds the maximum (toroidal Alfvén eigenmode) frequency, RSAEs cannot exist [10]. Toroidal rotation can significantly increase the GAM frequency, an effect that might be observable in spectrograms. In addition to such quantitative changes, rotation can also modify the structure of the spectrum. At very low frequencies, new flow-induced gaps and modes appear in the continuous spectrum $[16,17]$.

Whether cluster modes exist or not, depends both on whether certain cluster criteria are met and whether a free energy source is available to destabilize the modes. A radially decreasing density of large orbit energetic particles can play a role in satisfying both these conditions. They were shown to favour the existence of modes with a ratio of poloidal and toroidal mode numbers $m / n$ slightly above the minimum value $q_{0}$ of the safety factor $q$ [6]. Typically in experiments $q_{0}$ is decreasing due to current diffusion so that these modes chirp upwards in frequency. Second order toroidal effects were found to further favour upsweeping cascades [18]. Simulations showed that plasma pressure gradients provide an additional drive [19]. Terms quadratic in the pressure gradient were found to play a negative role for the existence of RSAEs [15]. Inclusion of the averaged normal curvature from the interchange term resulted in a mode existence criterion 
that, close to a rational surface, is similar to Mercier's stability criterion [20]. This criterion contains a term linear in the pressure gradient that is favourable for both stability and RSAE existence when $q>1$.

Here we extend these useful existing cluster criteria to take into account the effect of toroidal rotation. The results are limited to modes near a rational surface, where the mode frequency is close to the GAM frequency. New terms due to rotation appear, the physical origin of which was discussed in [21].

\section{Preliminaries}

\subsection{Assumptions}

We assume a tokamak plasma with a circular cross-section with minor radius $a$, centred at a major radius $R$ given by $R_{0}$. The nested magnetic surfaces, labelled by the poloidal flux function $\psi$, are assumed to be of constant equilibrium temperature $T(\psi)=p / \rho$ with $p$ and $\rho$ the plasma pressure and density. Within the magnetic surfaces, rotating with angular frequency $\Omega(\psi)$, the force balance $\partial p /\left.\partial R\right|_{\psi}=\rho R \Omega^{2}$ is then satisfied by $[22,23]$

$$
\frac{p(R, \psi)}{p_{\mathrm{s}}(\psi)}=\frac{\rho(R, \psi)}{\rho_{\mathrm{s}}(\psi)}=\exp \left(\frac{\mathcal{M}^{2} R^{2}}{R_{0}^{2}}\right),
$$

where $\mathcal{M} \equiv R_{0} \Omega / \sqrt{2 T}$ differs from a Mach number by a factor $\sqrt{\gamma / 2}$. The functions $p_{\mathrm{s}}(\psi)$ and $\rho_{\mathrm{s}}(\psi)$ represent the static pressure and density and can be freely specified.

We consider an infinitesimal plasma perturbation

$$
\boldsymbol{\xi}=\sum_{m^{\prime}=m, m \pm 1} \epsilon^{\left|m^{\prime}-m\right|} \boldsymbol{\xi}_{m^{\prime}}(r) \mathrm{e}^{\mathrm{i}\left(m^{\prime} \theta-n \phi-\omega t\right)}
$$

where higher order terms are only required at intermediary stages of the calculations [24]. The ordering of the sidebands with $\epsilon^{\left|m^{\prime}-m\right|}$ reflects the weak toroidal coupling to a dominant main perturbation $\boldsymbol{\xi}_{m}$. This ordering excludes the description of e.g. TAEs, which requires two harmonics to be dominant. A flux coordinate system $(r, \theta, \phi)$ is used in which, within magnetic surfaces labelled by $r$, the field lines have a constant pitch $\mathrm{d} \phi / \mathrm{d} \theta=q(r)$. In [24], a large aspect ratio expansion of the Frieman-Rotenberg equations [25] was performed up to fourth order in $\epsilon \equiv a / R_{0} \ll 1$, using the ordering

$\omega \sim \Omega, \quad \mathcal{M} \sim 1, \quad \beta \sim \epsilon^{2} \quad$ and $m / q-n \sim \epsilon$.

Here $\beta(r) \equiv 2 \mu_{0} p_{\mathrm{s}} / B_{0}^{2}$, where the constant $B_{0}$ refers to the magnetic field at $R=R_{0}$. With $\omega_{\mathrm{A}}(r) \equiv B_{0} / \sqrt{\mu_{0} \rho_{\mathrm{S}}} R_{0}$, (3) gives $\omega \sim \epsilon \omega_{\mathrm{A}}$, consistent with excluding the description of TAEs. The resulting mode equation will be discussed next.

\subsection{Mode equation}

The equation of motion for the radial component $\xi \equiv \xi_{m}^{r}$ derived in [24] can be written as

$$
\left(f \xi^{\prime}\right)^{\prime}+g \xi=h,
$$

where

$$
\begin{aligned}
& f=r^{3} A_{1}, \\
& g=r^{2} A_{2}^{\prime}-r\left(m^{2}-1\right) A_{1} .
\end{aligned}
$$

The function $h$ represents the coupling to the sideband harmonics $\xi_{m \pm 1}^{r}$ [24], a prime denotes $\mathrm{d} / \mathrm{d} r$, and

$$
\begin{gathered}
A_{1}=\tilde{A}_{1}+\left(\frac{m}{q}-n\right)^{2}, \\
A_{2}=\tilde{A}_{1}-n^{2}\left(\beta\left(1-q^{2}\right)+q^{2} \alpha_{k}+q^{2} \beta_{k}\right),
\end{gathered}
$$

where

$$
\begin{aligned}
\tilde{A}_{1} & =\frac{\left(\omega_{\mathrm{D}}^{2}-\tilde{\omega}_{-}^{2}\right)\left(\omega_{\mathrm{D}}^{2}-\tilde{\omega}_{+}^{2}\right)}{\omega_{\mathrm{A}}^{2}\left(\omega_{0}^{2}-\omega_{\mathrm{D}}^{2}\right)}, \\
\alpha_{k} & =\frac{\omega_{0}^{2}}{\omega_{\mathrm{A}}^{2}} \frac{4 \Omega \omega_{\mathrm{D}} / n}{\omega_{\mathrm{D}}^{2}-\omega_{0}^{2}}\left(1+\frac{\mathcal{M}^{2}}{\gamma}\right), \\
\beta_{k} & =\beta \mathcal{M}^{2}\left(1+\mathcal{M}^{2}\right) .
\end{aligned}
$$

The Doppler-shifted frequency $\omega_{\mathrm{D}}(r) \equiv \omega+n \Omega$ and

$\tilde{\omega}_{ \pm}^{2}(r)=\frac{\omega_{0}^{2}}{2}\left(\tilde{a}_{1} \pm \sqrt{\tilde{a}_{1}^{2}-\tilde{a}_{2}}\right) \quad$ and $\quad \omega_{0}^{2}(r)=\frac{\gamma T}{q^{2} R_{0}^{2}}$,

where

$$
\begin{aligned}
& \tilde{a}_{1}=1+2 q^{2}\left(1+\frac{1}{\gamma}\left(4 \mathcal{M}^{2}+\mathcal{M}^{4}\right)\right), \\
& \tilde{a}_{2}=\frac{8 q^{2}(\gamma-1)}{\gamma^{2}} \mathcal{M}^{4} .
\end{aligned}
$$

In the derivation of (4) in [24] the ordering $m / q-n \sim \epsilon$ was invoked to neglect radial variation in the safety factor. Therefore in all of the above expressions, except $(m / q-n)^{2}$, $q$ refers to its value $m / n$ at the rational surface. This actually requires an additional assumption of low magnetic shear. This is appropriate for the modes near a point of shear reversal as considered here.

\subsection{Continuous spectrum}

The frequencies of (8) satisfy $\tilde{\omega}_{-}^{2} \leqslant \omega_{0}^{2} \leqslant \tilde{\omega}_{+}^{2}$. The continuous spectrum is given by those frequencies for which $A_{1}=0$ so that (4) is singular. These continuum frequencies are given by $\omega_{\mathrm{D}}^{2}=\omega_{ \pm}^{2}$, where

$$
\omega_{ \pm}^{2}=\frac{\omega_{0}^{2}}{2}\left(a_{1} \pm \sqrt{a_{1}^{2}-a_{2}}\right) .
$$

Here

$$
\begin{aligned}
& a_{1}=\tilde{a}_{1}+\frac{\omega_{\mathrm{A}}^{2}}{\omega_{0}^{2}}\left(\frac{m}{q}-n\right)^{2}, \\
& a_{2}=\tilde{a}_{2}+4 \frac{\omega_{\mathrm{A}}^{2}}{\omega_{0}^{2}}\left(\frac{m}{q}-n\right)^{2},
\end{aligned}
$$

so that

$$
A_{1}=\frac{\left(\omega_{\mathrm{D}}^{2}-\omega_{-}^{2}\right)\left(\omega_{\mathrm{D}}^{2}-\omega_{+}^{2}\right)}{\omega_{\mathrm{A}}^{2}\left(\omega_{0}^{2}-\omega_{\mathrm{D}}^{2}\right)} .
$$

The toroidal flow-induced continuum gap for $-\tilde{\omega}_{-}<\omega_{\mathrm{D}}<$ $\tilde{\omega}_{-}$is caused by the centrifugal convective effect, where $\tilde{\omega}_{-}$is similar to a buoyancy or Brunt-Väisälä frequency $[16,23]$. 
The frequency $\tilde{\omega}_{+}$is known as the GAM frequency or beta-induced Alfvén eigenmode (BAE) frequency, and marks the minimum frequency of RSAEs. Neglecting the convective effect by assuming $\tilde{a}_{2} \ll \tilde{a}_{1}^{2}$, we obtain $\tilde{\omega}_{+} \approx \sqrt{\tilde{a}_{1}} \omega_{0}$. The coefficient $\tilde{a}_{1}$ represents the toroidal inertial enhancement factor for incompressible poloidal (Pfirsch-Schlüter) motion within a magnetic surface.

\section{Analysis}

\subsection{Oscillation properties}

Sturm's fundamental oscillation theorem with the modification due to Picone [26] states that in a given interval the homogeneous solution $\xi_{2}$ of (4) has the same number of nodes or more than $\xi_{1}$ when

$$
f_{1} \leqslant f_{2}<0 \quad \text { and } \quad g_{1} \geqslant g_{2} .
$$

Since RSAEs have frequencies $\omega_{\mathrm{D}}^{2} \geqslant \omega_{+}^{2}$, from (12) we find that $f=r^{3} A_{1} \leqslant 0$. When $\alpha_{k}^{\prime}$ can be neglected, for example for high $m, f$ and $g$ of (5b) satisfy conditions (13) when $\omega_{\mathrm{D} 2}^{2}<\omega_{\mathrm{D} 1}^{2}$. Therefore, the number of nodes of these solutions increases with decreasing $\omega_{\mathrm{D}}^{2}$. This is in agreement with simulations and observations of higher harmonics of RSAEs [27, 28].

More generally, when $\alpha_{k}^{\prime}$ can be neglected, the theorem implies that $\omega_{\mathrm{D}}^{2}$ increases with the number of nodes (Sturmian) when $A_{1}>0$ and decreases when $A_{1}<0$ (anti-Sturmian). This agrees with the finding of Sturmian sequences of modes in the frequency ranges $\omega_{\mathrm{D}}^{2} \leqslant \omega_{-}^{2}$ and $\omega_{0}^{2} \leqslant \omega_{\mathrm{D}}^{2} \leqslant \omega_{+}^{2}$ in [21] and [17], respectively. Note that we used here a mathematical argument since no analogue exists for rotating toroidal plasmas to the physics-based Goedbloed-Sakanaka oscillation theorem for static cylindrical plasmas [29].

\subsection{Time variation}

For high poloidal mode numbers $m$, the time variation of $q$ in the field line bending terms $(m / q-n)^{2}$ dominates the time variation of $\omega_{+}$so that from (10)-(11b)

$$
\frac{\mathrm{d} \omega_{+}}{\mathrm{d} t}=\frac{\omega_{\mathrm{A}}^{2}}{\omega_{+}} \frac{\omega_{+}^{2}-\omega_{0}^{2}}{\omega_{+}^{2}-\omega_{-}^{2}}\left(\frac{m}{q}-n\right) m \frac{\mathrm{d} q^{-1}}{\mathrm{~d} t} .
$$

When $q$ decreases and drops below $m / n$, there is an initially slow but accelerating increase in $\omega_{+}$, explaining the hockey-stick-shaped spectral lines of RSAEs observed in spectrograms $[3,15,28,30]$. Measured slopes $\mathrm{d} \omega_{+} / \mathrm{d} t$ can be used to discriminate between different poloidal mode numbers $m$ and obtain the time variation of the $q$ profile.

At the low-frequency tip of these spectral lines, when $q=m / n$ so that $\omega_{+}=\tilde{\omega}_{+}$, we have

$\frac{\mathrm{d} \omega_{+}}{\mathrm{d} t}=\frac{\omega_{+}}{\omega_{0}} \frac{\mathrm{d} \omega_{0}}{\mathrm{~d} t}+\frac{\omega_{0}^{2}}{\omega_{+}^{2}-\omega_{-}^{2}}\left(\frac{\omega_{+}}{2} \frac{\mathrm{d} \tilde{a}_{1}}{\mathrm{~d} t}-\frac{\omega_{0}^{2}}{8 \omega_{+}} \frac{\mathrm{d} \tilde{a}_{2}}{\mathrm{~d} t}\right)$.

When $\tilde{a}_{2} \ll \tilde{a}_{1}^{2}$ this approximately reduces to $\mathrm{d} \sqrt{\tilde{a}_{1}} \omega_{0} / \mathrm{d} t$. In spectrograms the continuum frequency $\omega_{+}$itself is generally not visible. The global RSAEs with approximately the same frequency, however, can be observed. The goal of the following analysis will be to establish under which conditions RSAEs can cluster above the $\omega_{+}$continuum.

\subsection{Cluster point analysis}

The nature of the differential equation (4) changes when at a radial position where $x \equiv r-r_{\mathrm{c}}=0$ the 'Mercier index' $D$ satisfies [31,32]

$$
D \equiv \lim _{x \rightarrow 0} x^{2} \frac{g}{f}>\frac{1}{4} .
$$

In this case the local homogeneous solutions $x^{\nu_{ \pm}}$, with the complex 'Frobenius indices' $v_{ \pm}=-\frac{1}{2} \pm \sqrt{1-4 D}$, rapidly oscillate and diverge near $x=0$. This signals the presence of an infinite sequence of modes near the cluster point at $r=r_{\mathrm{c}}$. By investigating $D$ for (4) in plasmas with a reversed magnetic shear $\left(q^{\prime}<0\right)$, we obtain criteria for the existence of RSAEs near the point of shear reversal $\left(q^{\prime}=0\right)$.

We note that (16) can only be satisfied when $A_{1}=A_{1}^{\prime}=0$ and only the behaviour of $A_{1}$ to second order in $x$ is of importance. In this case (16) becomes

$$
D=\left.\frac{2 A_{2}^{\prime}}{r A_{1}^{\prime \prime}}\right|_{r=r_{\mathrm{c}}}>\frac{1}{4} .
$$

Because of the magnetic field line bending term in $A_{1}, A_{1}^{\prime \prime}$ will typically be positive. In this case (17) shows that the clustering of modes requires $A_{2}^{\prime}>0$. When $A_{2}^{\prime}<0$ potentially unstable modes cannot cluster below the $\omega_{-}$continuum, ensuring local stability [21].

When, however, at $r=r_{0}$ the safety factor has a minimum $q_{0}<m / n$, magnetic field line bending decreases away from $r=r_{0}$ so that $A_{1}^{\prime \prime}$ is negative (see figure 1). This allows, in locally stable plasmas with $A_{2}^{\prime}<0$, the appearance of RSAEs.

Note that with $A_{1}=A_{1}^{\prime}=0$ and $A_{1}^{\prime \prime}<0$, (12) implies that $\omega_{\mathrm{D}}^{2 \prime \prime}>\omega_{+}^{2^{\prime \prime}}$. This will allow the sequence of RSAEs with frequencies $\omega_{\mathrm{D}}^{2} \geqslant \omega_{+}^{2}$ to avoid strong overlap with the continuum and associated continuum damping, see figure 1(a). When $q_{0}$ decreases in time, field line bending will increase, as will the mode frequency according to (14). This explains why for decreasing $q_{0}$ typically upsweeping RSAEs are found.

\subsection{Reversed magnetic shear}

We consider a plasma with a minimum $q_{0}$ in the safety factor, close $\left(m / q_{0}-n \sim \epsilon\right)$ to a rational surface $q=m / n$. To second order in $r-r_{0}$ we can write $q=q_{0}+\frac{1}{2} q_{0}^{\prime \prime}\left(r-r_{0}\right)^{2}$ so that

$$
\left(\frac{m}{q}-n\right)^{2}=\left(\frac{m}{q_{0}}-n\right)^{2}-\frac{\left(r-r_{0}\right)^{2}}{\Delta r^{2}},
$$

where we introduced a squared shear length scale

$$
\Delta r^{2} \equiv \frac{q_{0}^{2}}{m q_{0}^{\prime \prime}} \frac{1}{m / q_{0}-n} .
$$

The parallel wave number $-\left(m / q_{0}-n\right) / R_{0}$ is positive when $q_{0}>m / n$, so that $(m / q-n)^{2}$ increases quadratically away from $r=r_{0}$. When, however, $q_{0}<m / n$, field line bending decreases away from $r=r_{0}$ up to the rational surface $q=m / n$, after which it increases again. This causes the 'double well' of figure 1(b), the description of which requires the inclusion of terms of higher order in $r-r_{0}$. 
Note that in this case the continuum frequencies $\omega_{ \pm}^{2}$ have a similar double well shape, see figure 1(a). Sufficiently localized modes may therefore avoid strong continuum damping.

The cluster point is characterized by the two conditions $A_{1}=0$ and $A_{1}^{\prime}=0$. These are satisfied when either $\omega_{\mathrm{D}}=\omega_{+}$ and $\omega_{\mathrm{D}}^{\prime}=n \Omega^{\prime}=\omega_{+}^{\prime}$ as in figure $1(a)$ or $\omega_{\mathrm{D}}=-\omega_{+}$and $\omega_{\mathrm{D}}^{\prime}=n \Omega^{\prime}=-\omega_{+}^{\prime}$. When these criteria cannot be satisfied, RSAEs cannot exist. The expression for $A_{1}^{\prime \prime}$ at $r=r_{\mathrm{c}}$,

$$
A_{1}^{\prime \prime}=\left(\tilde{A}_{1}+(m / q-n)^{2}\right)^{\prime \prime}=2 \frac{N-1}{\Delta r^{2}},
$$

is naturally split into a term from (18) due to field line bending, and other effects due to $\tilde{A}_{1}^{\prime \prime}$ incorporated in $N$ :

$$
\begin{aligned}
N & \equiv \frac{\Delta r^{2}}{2} \frac{\tilde{\omega}_{+}^{2}-\tilde{\omega}_{-}^{2}}{\omega_{\mathrm{A}}^{2}\left(\omega_{0}^{2}-\tilde{\omega}_{+}^{2}\right)}\left(\omega_{\mathrm{D}}^{2}-\tilde{\omega}_{+}^{2}\right)^{\prime \prime}, \\
& \approx \Delta r^{2} \frac{\tilde{a}_{1}^{3 / 2}}{1-\tilde{a}_{1}} \frac{\omega_{0}}{\omega_{\mathrm{A}}} \frac{n \Omega^{\prime \prime}-\tilde{\omega}_{+}^{\prime \prime}}{\omega_{\mathrm{A}}},
\end{aligned}
$$

where in the second line we assumed $\tilde{a}_{2} \ll \tilde{a}_{1}^{2}$ and used (8). Note that in writing (20) we assumed that $r_{\mathrm{c}}$ is sufficiently close to $r_{0}$ that higher order terms in (18) can be neglected, for example $\left|r_{\mathrm{c}}-r_{0}\right| \ll q_{0}^{\prime \prime} / q_{0}^{\prime \prime \prime}$.

\subsection{Existence criterion for RSAEs}

Using (20), the cluster condition (17) is given by

$$
D=\frac{A_{2}^{\prime}}{N-1} \frac{\Delta r^{2}}{r}>\frac{1}{4} .
$$

As discussed previously, RSAEs can avoid strong continuum damping when $A_{1}^{\prime \prime}<0$. In this case the existence condition (22) is given by

$$
(1-N) \frac{r}{4 \Delta r^{2}}<-A_{2}^{\prime} .
$$

Therefore $-A_{2}^{\prime}$ should be sufficiently large and positive for the existence of RSAEs near their minimum frequency. With (6b) and (19) we can write this as

$$
(1-N)\left(\frac{m}{q_{0}}-n\right) \frac{r}{4} \frac{m}{n^{2}} \frac{q_{0}^{\prime \prime}}{q_{0}^{2}}<\beta^{\prime}\left(1-q^{2}\right)+q^{2} \alpha_{k}^{\prime}+q^{2} \beta_{k}^{\prime} .
$$

Equation (24) agrees with the previous result of $[19,20,27]$ that in a static plasma, where $\alpha_{k}=\beta_{k}=0$, a radially decreasing pressure is favourable for the existence of RSAEs provided that $q>1$. When, however, in a toroidally rotating plasma

$$
\mathcal{M}^{2}\left(1+\mathcal{M}^{2}\right)>1-1 / q^{2},
$$

the first and last terms on the right-hand side of (24) combined become negative. When this holds, a decreasing plasma pressure is unfavourable for RSAE existence.
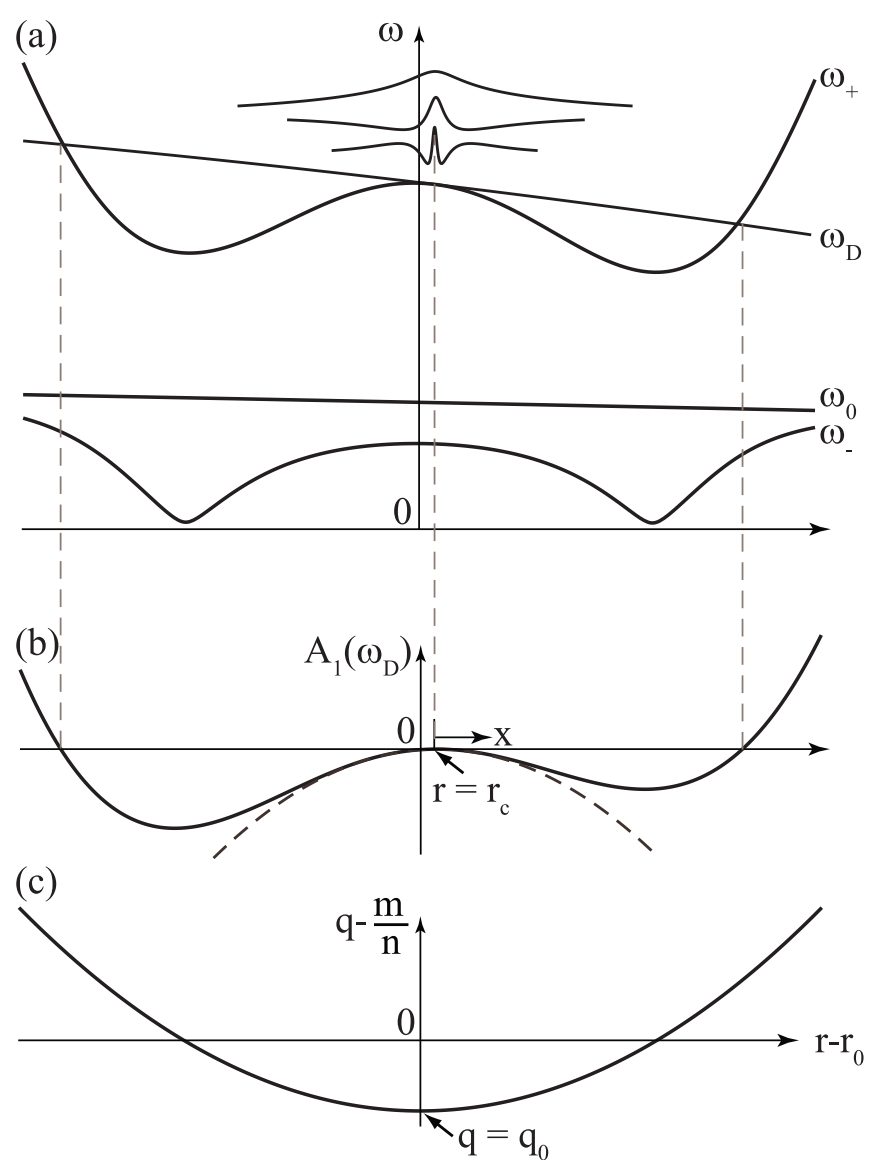

Figure 1. Schematic of the typical circumstances under which RSAEs cluster at a position $r=r_{\mathrm{c}}$ and frequency $(a) \omega_{\mathrm{D}}^{2}=\omega_{+}^{2}$ where $\left(\omega_{\mathrm{D}}^{2}\right)^{\prime}=\left(\omega_{+}^{2}\right)^{\prime}$ so that $(b) A_{1}=A_{1}^{\prime}=0$. This clusterpoint is not necessarily at the position $(c) r=r_{0}$ where the safety factor $q$ has its minimum $q_{0}$.

\subsection{Physical interpretation}

The first term on the right-hand side of (24) represents the well-known 'magnetic well'. It is the sum of the destabilizing pressure gradient and the stabilizing average toroidal field curvature.

For a large aspect ratio tokamak, using (1), $\boldsymbol{R}$. $\nabla \frac{1}{2} \rho R^{2} \Omega^{2} \approx \rho_{\mathrm{s}} R_{0}^{2} \Omega^{2}\left(1+\mathcal{M}^{2}\right)=\beta_{k} B_{0}^{2}$. Therefore $\beta_{k}$ is associated with the variation of the kinetic energy density with major radius. When $\beta_{k}^{\prime}<0$, the kinetic energy density decreases radially, which gives rise to instability analogous to the Rayleigh-Taylor instability of a fluid on top of a lighter fluid [21].

The term $\alpha_{k}$ is proportional to $\rho \Omega \omega_{\mathrm{D}}$ and its origin can be traced back to the Coriolis effect. For $\omega_{0}^{2}<\omega_{\mathrm{D}}^{2}$ and radially decreasing profiles, $\alpha_{k}^{\prime}$ is negative and thus unfavourable for the existence of modes with $\omega_{\mathrm{D}} / n>0$. From (2), the toroidal phase velocity $-\omega R / n$ is in this case smaller than the plasma velocity $R \Omega$. Observed from a frame locally moving along with the flow, these modes will move in the direction opposite to the plasma rotation. The effect is in this case favourable for modes with $\omega_{\mathrm{D}} / n<0$. Note that this term will be less significant for higher toroidal mode numbers $n$. 


\section{Experimental implications}

After shortly considering some effects lying outside the ideal MHD description used here, we will discuss the experimental implications of the present analysis.

\subsection{Adiabatic constant}

Since we apply MHD to a plasma that is not strongly collisional, we must allow for a value of the adiabatic constant $\gamma$ that deviates from the ideal gas value $\gamma=5 / 3$. For the description of RSAEs in a low- $\beta$ tokamak plasma this is likely to be an overestimate and a value of $\gamma \approx 1.4$ seems more appropriate [33]. Since $\sqrt{\gamma T}$ is the sound speed $c_{\mathrm{s}}$ in an ideal gas, we may write $\omega_{0}=c_{\mathrm{s}} / q R_{0}$ and replace $c_{\mathrm{s}}$ with a more general expression:

$$
c_{\mathrm{s}}^{2}=\frac{\sum_{j} \gamma_{j} n_{j} T_{j}}{\sum_{j} n_{j}},
$$

which is a sum over species $j$ including both electrons (e) and ions (i) with number densities $n_{j}$. For a monocomponent plasma with $\gamma_{\mathrm{e}}=1$ and $\gamma_{\mathrm{i}}=7 / 4$ we obtain $\omega_{0}^{2}=\left(T_{\mathrm{e}}+7 T_{\mathrm{i}} / 4\right) / q^{2} R_{0}^{2}$ as in [15]. Note that the definition of temperature used here includes a factor $k_{\mathrm{B}} / m_{j}$ with $k_{\mathrm{B}}$ Boltzmann's constant and $m_{j}$ the component's particle mass.

\subsection{Energetic ions}

Without rotation, $D$ of (22) equals $Q_{\mathrm{p} \text { (ressure) }}$ of [20,27] when evaluated near the rational surface at low frequency. We may follow the approach of $[18,15,20]$ and add independently derived toroidal and hot particle terms, $Q_{\text {tor }}$ and $Q_{\text {hot }}$, respectively, to obtain an extended criterion for mode existence. Near a rational surface $Q_{\text {tor }}$ is negligible compared with $Q_{\mathrm{p}}$ [20] and the expression of $Q_{\text {hot }}$ from [18] writes

$$
\frac{Q_{\text {hot }}}{D}=(1-N) \frac{\omega \omega_{\mathrm{h}}}{\omega_{\mathrm{A}}^{2}} \frac{\left\langle\rho_{\mathrm{h}}\right\rangle^{\prime} / \rho}{A_{2}^{\prime} / m} .
$$

In a tokamak $\omega_{\mathrm{A}}$ typically exceeds both the RSAE frequency $\omega$ and the hot ion gyrofrequency $\omega_{\mathrm{h}}$ by several orders of magnitude. Therefore, the flux surface averaged hot particle density $\left\langle\rho_{\mathrm{h}}\right\rangle$ has to be peaked and must be a significant fraction of the plasma density $\rho$ to compete with the plasma pressure and rotation effects of $A_{2}^{\prime} / m \sim \epsilon^{2}$.

\subsection{Frequency}

The primary effect of toroidal rotation on the RSAE frequency that is often observed is the Doppler shift $n \Omega$. It can be used to determine the mode numbers and the angular rotation frequency $\Omega$. For high $n$ it can even dominate the RSAE frequency [30].

In current tokamaks the Mach number can be a significant fraction of one. In JET for example, NBI heated plasmas can have $\mathcal{M} \approx 0.5$, see for example [34]. This approximately doubles $\tilde{a}_{1}$, causing an increase of about $40 \%$ in the minimum RSAE frequency $\tilde{\omega}_{+} \approx \sqrt{\tilde{a}_{1}} \omega_{0}$. Such a change should be observable.
Near the minimum RSAE frequency, when magnetic field line bending is still small, changes in temperature and the safety factor enter the frequency evolution according to (15). When, however, magnetic field line bending is the primary source of the RSAE frequency change, (14) shows that $\mathrm{d} \omega_{+}^{2} / \mathrm{d} t$ approximately scales with $\omega_{\mathrm{A}}^{2}$.

\subsection{Existence}

Recently, RSAEs have been observed between sawtooth crashes when the magnetic shear was very low and $q_{0} \approx 1$ [3]. Near $q=1$, the average magnetic well term $-\beta^{\prime}\left(1-q^{2}\right)$ in the existence criterion (24) is small. Shaping effects like ellipticity and triangularity are known to 'deepen' the magnetic well, making it vanishes at a somewhat lower $q$. In this case the rotational terms $\alpha_{k}^{\prime}$ and $\beta_{k}^{\prime}$ can be critical for RSAE existence in shots with significant toroidal rotation.

For RSAEs near a rational surface $\omega_{\mathrm{D}} \approx \pm \tilde{\omega}_{+} \approx \pm \sqrt{\tilde{a}_{1}} \omega_{0}$ so that

$$
\alpha_{k} \approx \pm \sqrt{8 \gamma} \frac{\sqrt{\tilde{a}_{1}}}{\tilde{a}_{1}-1} \frac{\beta \mathcal{M}}{m}\left(1+\frac{\mathcal{M}^{2}}{\gamma}\right) .
$$

With $\gamma=1.4, q=1$, and $\mathcal{M}=0.5$ this gives $\alpha_{k} \approx \pm 1.7 \beta / \mathrm{m}$. Typically both $\beta$ and $\mathcal{M}$ are decreasing functions of minor radius, so that $\alpha_{k}^{\prime}>0$ for RSAEs with $\omega_{\mathrm{D}} / m<0$. According to (24) a radially increasing $\alpha_{k}$ is favourable for mode existence. Therefore, modes with a toroidal phase velocity exceeding the plasma rotation velocity so that $\omega_{\mathrm{D}} / m<0$, are favoured. Modes with a toroidal phase velocity below the plasma rotation velocity receive a strong negative contribution to the right-hand side of the existence criterion (24). They may therefore not exist. From (28) this Coriolis effect is strongest for low $m$.

For $\mathcal{M}=0.5$, the centrifugal term $\beta_{k} \approx 0.3 \beta$ is a bit smaller, but does not decrease with $m$. From (27), it becomes more significant compared with fast particle effects for higher $m$. Typically $\beta_{k}^{\prime}<0$ so that from (24) it is always unfavourable for the existence of RSAEs. It should therefore be possible to see RSAEs in the GAM frequency range disappear with increasing rotation. First the modes moving slower than the plasma, lowest $-m$ modes first.

\subsection{Downwards sweeping cascades}

In the vast majority of cases, RSAEs are observed to increase in frequency with time. Occasionally, however, downward sweeping cascades are observed. In experiments with significant toroidal rotation, upwards sweeping RSAEs propagating in the direction opposite to the plasma rotation appeared to sweep downwards in frequency due to the Doppler shift $n \Omega$ [30]. In other cases the observation of downwards chirping cascades required the introduction of energetic particles, kinetic effects, and 'quasi-modes' to be explained $[6,35,36]$. Here we show that also within the present MHD framework there are circumstances under which downwards sweeping cascades can arise.

The typical situation for which upsweeping cascades occur is shown in figure 1 . When $q_{0}<m / n$ decreases, field line bending increases and the mode frequency shifts 
upwards. When, however, $q_{0}$ is above $m / n$, field line bending decreases when $q_{0}$ decreases, resulting in downwards sweeping cascades. The increasing field line bending away from $r=r_{0}$ will, however, generally lead to a local minimum in the continuum, instead of a maximum as in figure 1(a). The anti-Sturmian sequence of modes clustering above this continuum will unavoidably overlap in frequency with the continuum. This will lead to heavy continuum damping, providing an explanation for why these downwards sweeping cascades are rarely observed. Note that a Sturmian sequence of modes below the continuum with $\omega_{\mathrm{D}}^{2}<\omega_{+}^{2}$ (BAEs in regular shear plasmas) may still avoid continuum damping and sweep downwards.

RSAEs with frequencies $\omega_{\mathrm{D}}^{2}>\omega_{+}^{2}$ can thus avoid strong continuum damping when $A_{1}^{\prime \prime}<0$. Depending on $N$, there are several possibilities for this to happen, summarized by the following diagram:

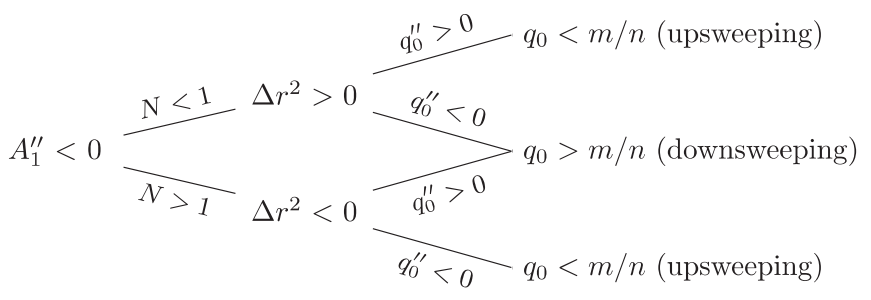

where the conclusions regarding upsweeping or downsweeping frequency assume decreasing $q_{0}(t)$. The first case with $N<1$ and a minimum in the safety factor profile, resulting in upwards shifting frequencies, is the typical case for RSAEs. The second case shows that strong continuum damping may also be avoided $\left(A_{1}^{\prime \prime}<0\right)$ when there is a local maximum $q_{0}$ in the safety factor profile $\left(q_{0}^{\prime \prime}<0\right)$ that exceeds $m / n$. These are precisely the conditions under which simulations showed down-chirping RSAEs [37]. Also in LHD experiments with $q_{0}^{\prime \prime}<0$, RSAEs with decreasing frequency were found [12].

The above diagram also shows another instance in which downsweeping may occur, namely when $N>1$. According to $(21 b)$ this can happen, for example when $n$ is high and the rotation frequency is locally concave $\left(\Omega^{\prime \prime}>0\right)$. In this case the Doppler shift ensures that, at least locally, $\omega_{\mathrm{D}}$ curves away from the continuum so that strong continuum damping can be avoided.

\subsection{Other modes}

Equation (22) is also a necessary condition for the existence of modes clustering above the $\omega_{-}$continuum. This would predict, at low frequencies very close to the Doppler shift, the possibility of a second cascade of RSAEs. For these low frequencies $\alpha_{k}^{\prime}$ is somewhat smaller and favours positive instead of negative Doppler-shifted frequency modes. Note that in this case, $N$ is obtained by interchanging $\omega_{+}$and $\omega_{-}$ in $(21 a)$.

For positive shear plasmas, (18) is replaced by $(m / q-$ $n)^{2}=\left(n q^{\prime} / q\right)^{2}\left(r-r_{0}\right)^{2}$. By replacing $\Delta r^{2}$ with $-\left(q / n q^{\prime}\right)^{2}$, therefore, (22) becomes a criterion for the existence of modes clustering below the $\omega_{ \pm}$continua of regular shear plasmas:

$$
\frac{r}{4}\left(\frac{q^{\prime}}{q}\right)^{2}<\frac{-\beta^{\prime}\left(1-q^{2}\right)-q^{2} \alpha_{k}^{\prime}-q^{2} \beta_{k}^{\prime}}{1-N} .
$$

This criterion improves on the derived integral condition for the flow driven 'BAEs' found in [17] by taking into account the effect of magnetic shear. When $\gamma=1$, the clusterfrequency $\tilde{\omega}_{-}=0$ is marginally stable. Modes clustering below this continuum are therefore automatically unstable. In this case, the reverse of (29) provides a stability criterion for localized (Mercier) modes [21].

\section{Discussion and conclusions}

We derived an existence condition, (24), for reversed shear Alfvén eigenmodes near a rational surface using a clusterpoint analysis. Toroidal plasma rotation enters through a centrifugal effect and an effect depending on the Doppler-shifted mode frequency. The latter Coriolis effect is typically unfavourable for RSAEs with a toroidal phase velocity that is below the plasma velocity or in the opposite direction. Neglecting this term, we showed that RSAEs form an anti-Sturmian sequence with their number of nodes decreasing with increasing frequency. We finally showed that the sometimes observed RSAEs sweeping downwards in frequency can avoid strong continuum damping when the safety factor contains a local maximum, or the flow profile is strongly concave.

We note that local existence criteria of the kind discussed here, neglect coupling between poloidal harmonics allowing the (weak) ballooning of a mode towards the low-field side of the plasma. This destabilizing ballooning effect is even stronger in the presence of rotation, as the kinetic energy adds to the static pressure in the coupling terms. When the magnetic shear is low enough for the sideband harmonics to be located away from their respective resonance, the effect of the coupling term $h$ of (4) will, however, be stabilizing [17]. The influence of this coupling on the derived existence criterion (22) for RSAEs is therefore somewhat non-trivial.

In addition to the influence on the existence of RSAEs, toroidal rotation also increases their clusterpoint frequency given by (10). Thereby, a changing toroidal rotation weakly enters the time variation of the RSAE frequency as described by (14) and (15). Effects associated with the compression of Alfvén waves are also included here. Taking all of these effects into account leads to improved predictions and a better understanding of the low-frequency MHD spectrum.

\section{Acknowledgments}

I thank H J de Blank, J P Goedbloed and B Koren for comments. This work, supported by NWO and the European Communities under the contract of the Association EURATOM/FOM, was carried out within the framework of the European Fusion Program. The views and opinions expressed herein do not necessarily reflect those of the European Commission.

Euratom @ 2012. 


\section{References}

[1] Goedbloed J P, Holties H A, Poedts S, Huysmans G T A and Kerner W 1993 Plasma Phys. Control. Fusion 35 B277

[2] Sharapov S E, Testa D, Alper B, Borba D N, Fasoli A, Hawkes N C, Heeter R F, Mantsinen M, Von Hellermann M G and contributors to the EFDA-JET work-programme 2001 Phys. Lett. A 289127

[3] Edlund E M, Porkolab M, Kramer G J, Lin L, Lin Y and Wukitch S J 2009 Phys. Rev. Lett. 102165003

[4] Heidbrink W W et al 2008 Nucl. Fusion 48084001

[5] Kimura H et al 1998 Nucl. Fusion 38 1303-14

[6] Berk H L, Borba D N, Breizman B N, Pinches S D and Sharapov S E 2001 Phys. Rev. Lett. 87185002

[7] Nazikian R, Kramer G J, Cheng C Z and Gorelenkov N N 2003 Phys. Rev. Lett. 91125003

[8] Classen I G J et al 2010 Rev. Sci. Instrum. 81 10D929

[9] van Zeeland M A, Kramer G J, Austin M E, Boivin R L, Heidbrink W W, Makowski M A, McKee G R, Nazikian R, Solomon W M and Wang G 2006 Phys. Rev. Lett. 97135001

[10] Fredrickson E D, Crocker N A, Gorelenkov N N, Heidbrink W W, Kubota S, Levinton F M, Yuh H, Menard J E and Bell R E 2007 Phys. Plasmas 14102510

[11] Gryaznevich M P et al 2008 Nucl. Fusion 48084003

[12] Toi K et al 2010 Phys. Rev. Lett. 105145003

[13] Winsor N, Johnson J L and Dawson J M 1968 Phys. Fluids $112448-50$

[14] Goedbloed J P 1975 Phys. Fluids 18 1258-68

[15] Breizman B N and Pekker M S 2005 Phys. Plasmas 12112506

[16] van der Holst B, Beliën A J C and Goedbloed J P 2000 Phys. Plasmas 7 4208-22

[17] Haverkort J W, de Blank H J and Koren B 2011 Plasma Phys. Control. Fusion 53045004

[18] Breizman B N, Berk H L, Pekker M S, Pinches S D and Sharapov S E 2003 Phys. Plasmas 10 3649-60
[19] Kramer G J, Gorelenkov N N, Nazikian R and Cheng C Z 2004 Plasma Phys. Control. Fusion 46 L23-L29

[20] Fu G Y and Berk H L 2006 Phys. Plasmas 13052502

[21] Haverkort J W and de Blank H J 2011 Plasma Phys. Control. Fusion $\mathbf{5 3} 045008$

[22] Maschke E K and Perrin H 1980 Plasma Phys. 22579

[23] Haverkort J W, de Blank H J and Koren B 2011 J. Comput. Phys. 231 981-1001

[24] Wahlberg C 2009 Plasma Phys. Control. Fusion 51085006

[25] Frieman E and Rotenberg M 1960 Rev. Mod. Phys. 32 898-902

[26] Ince E L 1956 Ordinary Differential Equations (New York: Dover)

[27] Gorelenkov N N, Kramer G J and Nazikian R 2006 Plasma Phys. Control. Fusion 48 1255-69

[28] van Zeeland M A et al 2009 Nucl. Fusion 49065003

[29] Goedbloed J P and Sakanaka P H 1974 Phys. Fluids 17 908-18

[30] Kramer G J et al 2006 Phys. Plasmas 13056104

[31] Bender C M and Orszag S A 1978 Advanced Mathematical Methods for Scientists and Engineers (New York: McGraw-Hill)

[32] Goedbloed J P and Poedts S 2004 Principles of Magnetohydrodynamics (Cambridge: Cambridge University Press)

[33] Edlund E M, Porkolab M, Kramer G J, Lin L, Lin Y, Tsujii N and Wukitch S J 2010 Plasma Phys. Control. Fusion 52115003

[34] Versloot T W et al 2010 Plasma Phys. Control. Fusion 52045014

[35] Gorelenkov N N 2008 Phys. Plasmas 15110701

[36] Breizman B N and Sharapov S E 2011 Plasma Phys. Control. Fusion 53054001

[37] Edlund E M, Porkolab M, Kramer G J, Lin L, Lin Y and Wukitch S J 2009 Phys. Plasmas 16056106 